Perspective

\title{
What ADME tests should be conducted for preclinical studies? Hong Wan
}

Shanghai Hengrui Pharmaceutical CO., LTD.

Dept. of DMPK/Tox, Shanghai, P.R. China

E-mail: wanh@shhrp.com

Tel: +86(0)21-54759066-1313; Fax: +86(0)21-54759072; Mobile: $+86-18036618308$

Received: June 06 ${ }^{\text {th }}, 2013$; Revised: July 01 ${ }^{\text {st }}, 2013$; Published: July $03^{\text {rd }}, 2013$

\begin{abstract}
The pharmaceutical industry has been evolving in recent years, which made numerous CROs and biotech companies conducting drug discovery and development programs and services in China. As a very comprehensive and important technology platform bridging efficacy and safety, the DMPK has become a mature discovery function to optimise ADME properties in drug design and screening, and dramatically mitgate attrition rates during the last decades. In this article, the author addresses several frequent questions associated with ADME/DMPK studies, e.g., what ADME tests should be conducted for preclinical studies? What should a typical investigational new drug (IND) enabling package cover? Which ADME/DMPK studies require good laboratory practice (GLP) or non-GLP? What does a good PK profile look like? The author presents a straightforward overview of these basic questions from his many years' experience in both pharmaceutical research and CRO in supporting drug discovery projects and IND filing.
\end{abstract}

\section{Keywords}

DMPK, PK, toxicity, safety, screening cascade, metabolite, drug-drug interaction, investigational new drug

\section{DMPK/ADME: definition and study purpose}

DMPK, or Drug Metabolism and Pharmacokinetics, is an important part of studies often referred to as ADME (Absorption, Distribution, Metabolism, and Elimination).

- Absorption (how much and how fast, often referred to as the absorbed fraction or bioavailability)

- Distribution (where the drug is distributed, how fast and how extensive)

- Metabolism (how fast, what mechanism/route, what metabolite is formed, and whether they are active or toxic)

- Elimination (how fast, which route).

In the drug discovery process, early in vitro ADME screening and in vivo PK profiling provide a basis for choosing new molecular entities (NMEs) and lead compounds that have desirable drug metabolism, PK or safety profiles, necessary for drug candidate selection (CS) and late-stage preclinical and clinical development. The ADME properties of a drug allow the drug developer to understand the safety and efficacy data required for regulatory approval. 
Today, DMPK studies performed in vitro and in vivo have become more standardised procedures across the pharmaceutical industry. A couple of typical examples include the most commonly used in vitro ADME studies, such as liver microsomes and the whole hepatocyte models for in vitro metabolism. Both models contain major metabolism enzymes, such as CYP450 for phase I metabolism and UDP-glucuronosyltransferase (UGT) for phase II metabolism. These relevant in vitro models are also applied to CYP inhibition/induction studies as well as drug metabolite identification and profiling across species. Other key in vitro assays, such as Caco-2 or MDCK cell-based models are often used for intestinal permeability evaluation. For in vivo PK studies, Association for Assessment and Accreditation of Laboratory Animal Care (AAALAC)-accredited animals, such as mice, rats, dogs, and non-human primates are employed to generate in vivo PK data like drug clearance $(\mathrm{CL})$, bioavailability $(\mathrm{F} \%)$, exposure $(\mathrm{AUC})$, half life $\left(t_{1 / 2}\right)$, and distribution volume $(L)$. Currently, almost all DMPK-related assays are carried out by available automated technology platforms combined with high-throughput liquid chromatography-mass spectrometry (LC/MS/MS) bioanalysis, which has considerably speeded up the ADME data generation for decision-making during the drug discovery and development process.

\section{What ADME tests should be conducted for preclinical studies?}

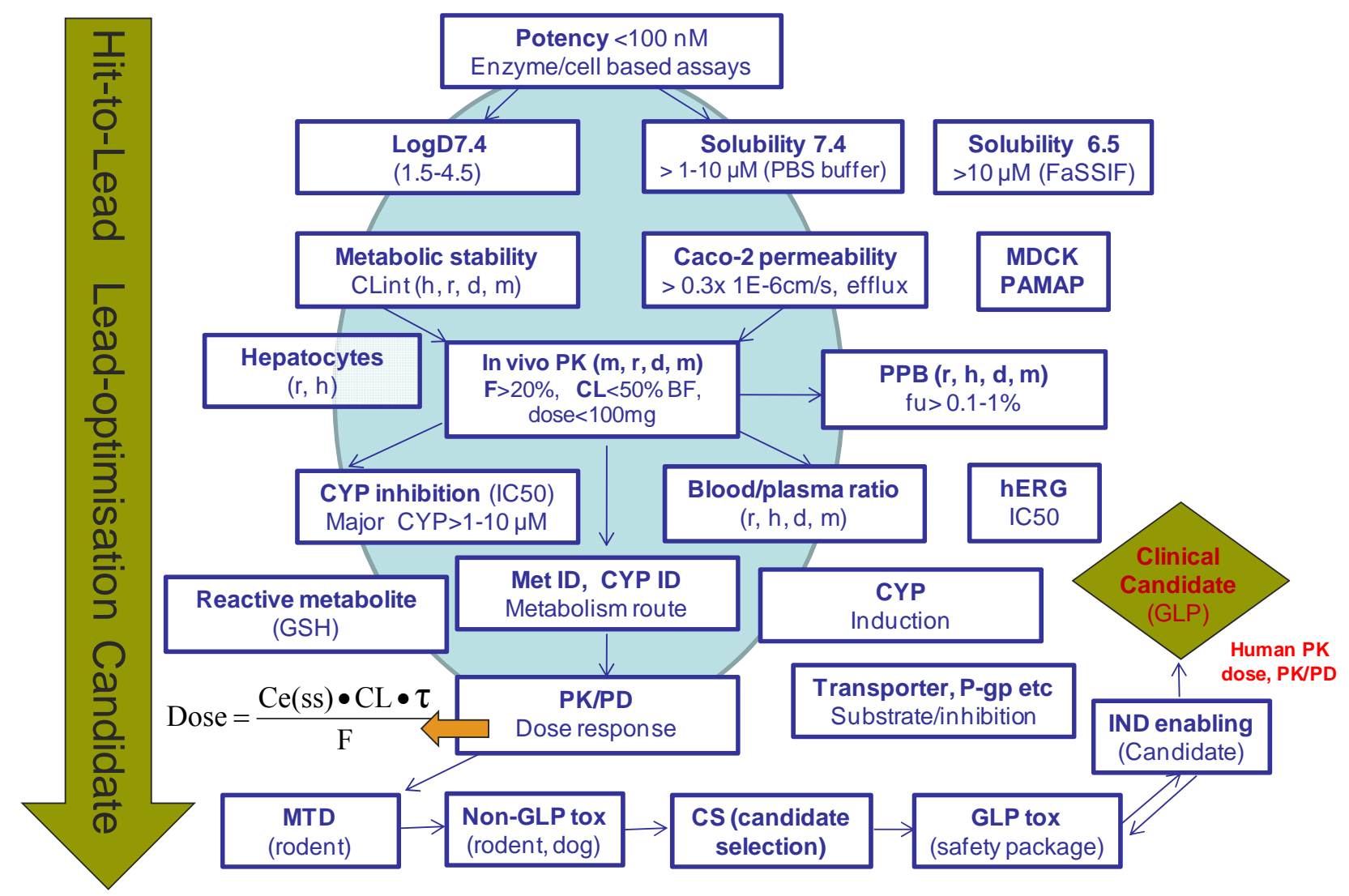

Figure 1: Typical ADME/PK screening cascade

Figure 1 highlights a typical ADME/DMPK screening cascade to support drug discovery programs from early hit-to-lead, to lead optimisation and candidate evaluations. As depicted in Figure 1, a number of DMPK studies are required before CS, followed by non-GLP and GLP toxicology studies for the IND-enabling package that is necessary for clinical candidate development. Various studies and assays should be conducted at different stages to address ADME issues and meet specific criteria to enable the project to 
move forward. It should be noted that these criteria, as exemplified in this cascade, are not fixed numbers or filters, and depend on other parameters as well as the specific therapeutic targets of different drug discovery programs. This cascade just demonstrates a traditional downstream of ADME/PK tests for a typical drug discovery programs before the IND-enabling stage. Often, at the early stage of hit-to-lead, key in vitro assays and studies, such as solubility, liver microsomal stability, CYP inhibition, and permeability should be conducted to provide key data for chemists to select the more metabolically stable and active/potent compounds for further in vivo PK studies. Solubility and $\log D$ data can serve as a basis to run various in vitro assays as well as for drug stability assessment and selection of suitable formulations. Also, it is well known that $\log D / \log P$ a crucial factor governing passive membrane partitioning, influencing permeability opposite to its effect on solubility. Moreover, if the in vitro metabolic stability data from phase I metabolism cannot predict in vivo hepatic plasma clearance, the phase II in vitro metabolism in hepatocytes and even hepatic transporters such as OATP1B1 are then necessary to find the relevant in vitro models for in vivo metabolism prediction to drive chemists to design and make more potent and selective compounds with improved PK profiles. Even at the very early stage of hit-to-lead, selective in vivo PK studies are valuable to confirm whether the applied in vitro assays (in vitro metabolism and absorption) can serve as good predictive models for in vivo PK in terms of plasma clearance and bioavailability. In some circumstances, if both in vitro liver microsomes and hepatocyte models might not predict well in vivo metabolism, more extensive in vivo PK studies have to be performed as a result of poor in vitro and in vivo correlations based on evaluations by a simplified well-stirred model. A clear structure-activity relationship (SAR)/ADME certainly facilitates the design of new compounds. For the drug absorption evaluations, there are three commonly used models, such as PAMPA, and Caco-2 and MDCK permeability. From the cost point of view, the PAMPA membrane permeability assay is the most cost-effective among these three in vitro models, with a fast turnaround, while the Caco-2 and MDCK-MDR1 models can offer more accurate absorption prediction as well as allow efflux and transporter studies for substrate and inhibition identification.

Traditionally, the CYP inhibition study is conducted at a late stage, e.g., from lead optimisation to CS. This has been now moved to earlier stages, even hit-to-lead. Table 1 shows a general decision tree on CYP inhibition based on drug-drug interaction (DDI) assessment, with an interpretation of potential for in vivo inhibition (derived from FDA Guidance for Industry: Drug Interaction Studies - Study Design, Data Analysis, Implications for Dosing, and Labeling Recommendations) [1]. An estimated [I]/Ki ratio of greater than 0.1 is considered positive and a follow-up in vivo evaluation is recommended. The likelihood of an in vivo interaction is projected based on the [I]/Ki ratio where [I] represents the mean steady-state $C_{\max }$ value for total drug (bound plus unbound) following administration of the highest proposed clinical dose. As the ratio increases, the likelihood of an interaction increases. Also, for CYP3A inhibition, two structurally unrelated substrates should be evaluated in vitro. If one of the two evaluations suggests a potential interaction (i.e., [I]/Ki of more than 0.1), an in vivo evaluation should be carried out. At the earlier screening stage, $I_{50}$ by a single-point screening assay, which is a more cost-effective approach with acceptably good correlation with full curve $I_{50}$ assay, can be used for initial DDI evaluation. A full curve $I_{50}$ or Ki determination can be utilised for DDI assessment at a late phase for candidates such as NMEs. Although quantitative predictions of in vivo DDI from in vitro studies are not possible, rank order across the different CYP enzymes for the same drug may help prioritise in vivo DDI assessment. 
Table 1: Prediction of clinical relevance of competitive CYP inhibition

\begin{tabular}{c|c|c}
\hline Likelihood & $\mathrm{I} \mathrm{C}_{50}(\mu \mathrm{M})$ & {$[\mathrm{I}] / \mathrm{Ki}[1]$.} \\
\hline Likely & $<1$ & $>1$ \\
Possible & $1<\mathrm{IC} 50<10$ & $0.1<[\mathrm{I}] / \mathrm{Ki}<1$ \\
Unlikely & $>10$ & $<0.1$ \\
\hline [I] is the concentration of inhibitor exposed to the active site of the enzyme in vivo, $\mathrm{Ki}$ is the inhibition \\
constant, which can be obtained by a full curve $\mathrm{IC}_{50}$ assay, or roughly estimated from the $\mathrm{IC}_{50}$, i.e., \\
\multicolumn{2}{c}{$\mathrm{Ki} \approx \mathrm{IC} 50 / 2$}
\end{tabular}

Similar studies associated with transporters are needed to identify whether the NMEs could be a potential substrate or inhibitor for P-gp or other important transporters for DDI as early as possible. More complex decision-making trees and recommended models for transporter-mediated DDI assessment are outlined in the FDA's new guidance on drug interaction studies. It should be pointed out that the FDA has extended DDI guidance from the 2006 draft, with 52 pages, to the current 75 pages (issued in 2012), reflecting the importance and complexity of drug interaction studies among the DMPK $[1,2]$. This is attributed to the high percentage of drug failures caused by DDI.

Another important aspect includes the drug metabolite identification and profiling as well as metabolism pathways in connection with CYP metabolism enzyme identification (the so-called CYP ID). Nowadays, the rapid metabolite identification (MetID) at a very early stage has also become relatively easy, such as with Metabolynx-assisted structure identification software, which is especially necessary for metabolically unstable compounds, whose biotransformation can help chemists understand the site of metabolic liability, for further structure modification for a desirable metabolism profile. With the issuance of the FDA's final guidance for industry on safety testing of drug metabolites (MIST), there is increased concern for obtaining metabolite data as early as possible in preclinical studies. The discovery of disproportionate drug metabolites late in drug development can potentially cause development and market delays. As guided in MIST published in 2008 as well as in the ICH M3 (R2) published in 2009 [3,4], if a metabolite is a unique human metabolite, or more commonly, if a circulating metabolite is present at disproportionately higher levels in humans than in the animal species used in toxicology studies, additional non-clinical safety assessment studies may be required. As exemplified in Figure 2, any metabolites found to be $>10 \%$ (based on systemic exposure at steady state) of total drug-related material should then be monitored in repeating dose animal toxicology studies to determine if they are present at higher levels in other species as well. Exceptions might be possible for lower-risk metabolites, such as most phase II conjugated metabolites. If the major metabolite exposure in at least one species is greater than in the human, then no additional safety studies of the metabolite are needed. It should be noted that the metabolite decision tree shown in Figure 2 is only a suggestion or recommendation, with an approximate $10 \%$ cut-off, which does not necessarily mean a metabolite testing safety guarantee. For instance, if a drug shows a low predicted efficacy dose (10 mg or lower), a metabolite found to be over $10 \%$ of total drug-related material might not require further safety tests. In contrast, for a highly dosed compound, even if a metabolite is lower than $10 \%$ of total drug-related material, it could be toxic, especially with accumulation of the metabolite. Also, a reactive metabolite might be toxic at a very low concentration. Overall, a metabolite safety testing decision should consider many factors, such as in vitro-in vivo correlations and especially in vivo animal toxicology findings, e.g., abnormalities or even animal deaths with repeat doses on animal studies. In this case, it is essential to identify the biotransformed metabolites to understand whether the observed toxicity is due to the accumulation of parent exposure or toxic metabolites, as even metabolites at a level lower than $10 \%$ 
can accumulate. Such additional toxicity testing of the major metabolite can be informative to confirm whether the cause of toxicity is the metabolite or not. In this case, for rodent or acute rat toxicology, the identification and profiling of drug metabolites using toxicokinetic plasma samples can be used. In short, relevant studies must be carried out to determine if human metabolites are adequately evaluated during non-clinical safety studies.

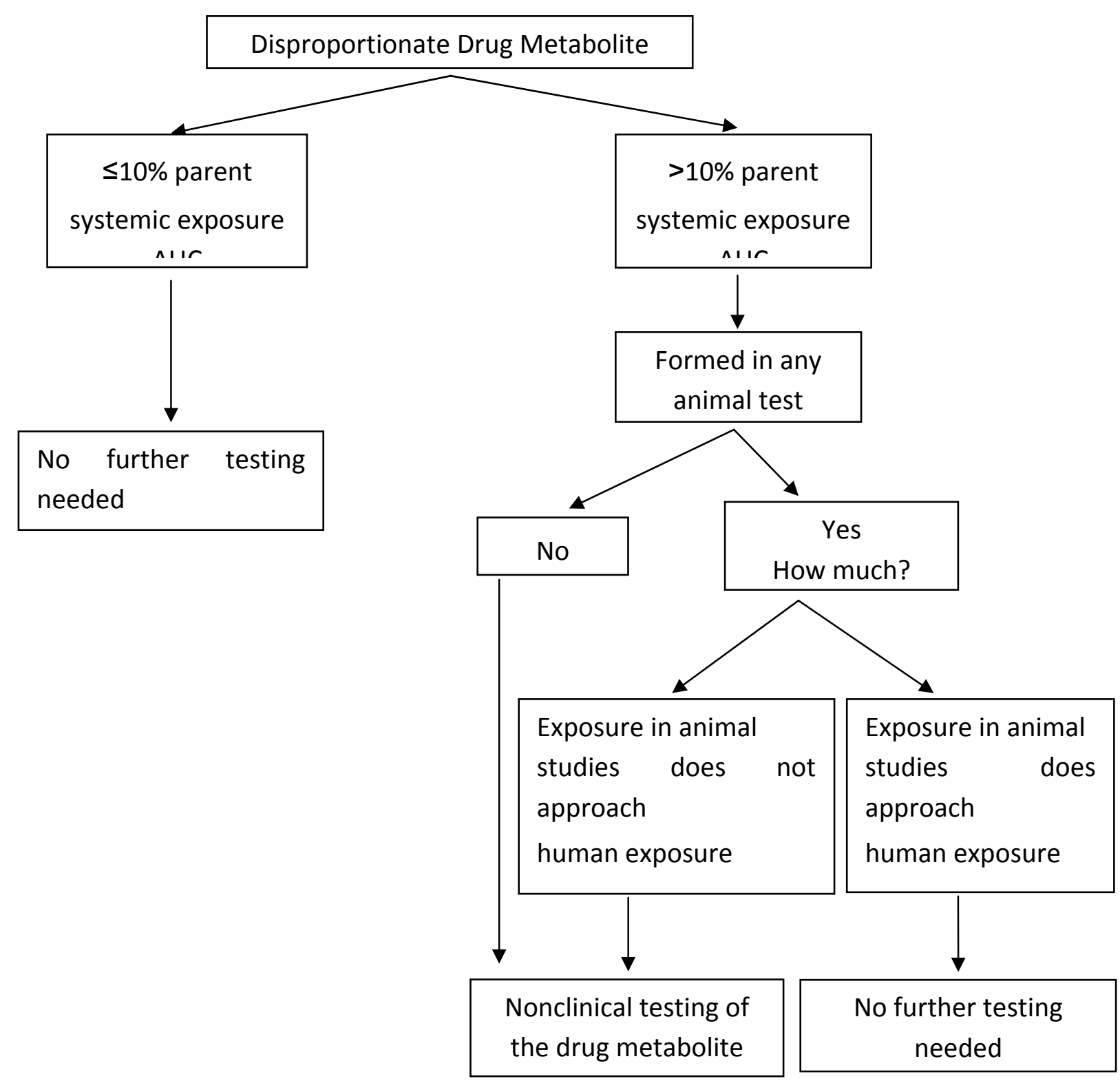

Figure 2: FDA suggested metabolite decision tree flow diagram

In addition to the CYP inhibition/induction and transporter studies associated with DDI, other safety-related studies, such as reactive metabolism and hERG should be performed as early as possible. The hERG inhibition test for cardiotoxicity has been shown to predict increased risk of arrhythmia, which can be initially evaluated under non-GLP condition by in vitro approaches that are cost-effective and have a fast turnaround, and later confirmed using QT interval prolongation measurements according to FDA guidelines [5]. Another important in vivo study is basic safety tests that are most commonly performed on rats and dogs, e.g., non-GLP acute toxicology (7-day or 14-day rat and dog repeat dose) for oral drugs, which usually include body weight, clinical chemistries, haematology, and histopathology (high dose and control only if no 
pathology is seen at the high dose). This study should be designed to establish a dose that induces a minimal toxic effect, or alternatively, to establish a safety margin (e.g., 10-100x depending on the different therapeutic targets). The selection of dose levels for repeat oral dose in toxicology studies is generally based on the minimum efficacy dose (MED) and the maximum tolerated dose (MTD). Based on the measured AUC (exposures) and toxicokinetic data, an approximate safety margin and compound accumulation characteristics can be known, which are paramount for the decision-making on whether to follow up a GLP-tox design or not. Such non-GLP studies help to identify more accurate dose level design for the subsequent GLP studies.

In summary, a comprehensive nonclinical ADME/PK package, including key toxicity data, should be generally complete by the time of CS. The ultimate goals of all ADME/PK/tox studies before the CS stage are to better understand the compound's metabolite-mediated toxicity and safety profile to make a concrete decision for the purpose of enabling IND, which is a crucial part of the drug approval process.

\section{IND-enabling package for GLP or non-GLP?}

What preclinical studies should be conducted to enable an IND? Should all studies be performed in GLP laboratories for IND filing? Some researchers are mis-communicating that at IND stage, all studies should be conducted in GLP laboratories. Actually, the FDA requires GLP documentation only for safety-related in vivo studies like GLP-tox. In other words, in vitro ADME and in vivo PK studies do not need to be performed in GLP labs. In fact, from a bioanalytical data quality point of view, there is no apparent discrepancy between non-GLP and GLP laboratories regarding bioanalytical procedures, because most non-GLP bioanalysis methods are performed according to FDA bioanalysis guidance [6]. The major difference can be in the study design, e.g., a typical non-GLP tox study utilises a small group of animals (three or four per group), while a GLP-tox study usually utilises more than 10 animals per group with half males and half females in one study. In addition, more strict documentation must be recorded for the purpose of regulatory compliance. Obviously, the GLP study has much higher costs than a similar non-GLP study, which is not recommended before CS for the IND-enabling package. Key components of the IND data package include pharmacology, toxicology and safety pharmacology, ADME and chemistry, and the manufacturing and control sections of the submission. A typical IND-enabling ADME package contains data from the following studies: bioanalytical method validation in one rodent and one or more non-rodent species; single- and multiple-dose PK; dose proportionality and absolute bioavailability in one rodent and one or more non-rodent species; and in vitro CYP inhibition/induction in human liver microsomes for DDI assessment (including likely transporter studies). Other data, such as mass balance and routes of excretion in rodents, including bilary excretion in rats, metabolite profiling and identification in rodents and non-rodents, tissue distribution using radio-labelled compound by quantitative whole-body autoradiography (QWBA) form part of the surrogate end-points, although these studies are not required for submission. Overall, complete ADME and toxicology data can make the submission package more compelling to move a compound into phase I clinical studies. Knowing the objectives, expectations, and processes of assembling and filing an IND is the key factor not only for a successful filing, but it can also accelerate a promising clinical development path forward. More detailed information on the general requirements and strategies for successful IND filings can be found in FDA's guidance [7], which is beyond the scope of the current main topic. In order to reduce the potential development cost, exploratory IND (eIND) preclinical studies are recommended by the FDA [8]. Such eIND studies are conducted prior to the traditional dose escalation, safety, and tolerance studies that ordinarily initiate a clinical development program, which is less extensive than for traditional IND studies. Overall, eIND studies provide the opportunity to already obtain human data in the drug discovery phase, such as important information on PK, PK/PD, and basic toxicology data for a key decision 
for selecting the most promising lead product. Even a microdose approach (e.g., $<100 \mu \mathrm{g}$, or $<1 / 100$ of MED) in a human study, using an advanced technology such as accelerator mass spectrometry (AMS) combined with radio-labelled compound can be very beneficial to gain human PK information as early as possible for IND enabling [9].

\section{What is a good PK profile?}

Another frequent question asked by drug discovery scientists is 'Is this compound's PK good?' Ideally, the desirable PK profiles of a preclinical candidate that is being considered for taking into development should:

- have acceptable solubility for development;

- be completely absorbed, preferably via passive absorption;

- have high bioavailability (e.g., $\mathrm{F}>50 \%$ ) for oral drug;

- have a low plasma clearance $\mathrm{CL}$ (e.g, $<30 \%$ blood flow), long half-life $\left(t_{1 / 2}\right)$ (e.g., $>6 \mathrm{hrs}$ ), and acceptable distribution volume;

- have a linear kinetics, i.e., exposure proportional to dose and a clear PK/PD correlation;

- be eliminated by several pathways, i.e., renal excretion and hepatic metabolism, also metabolised preferably by more than one enzyme for de-risking DDI;

- have a simple metabolite profile, with no reactive metabolite;

- have no obvious CYP and major transporters like P-gp inhibition or induction or low DDI potential; and

- have a sufficient or at least acceptable safety margin (safety margin $>10 x$, depending on different therapeutic targets).

In reality, we can readily rank which compound's PK is better than others from the same series of compounds during the screening. However, we should not rule out a compound by only looking at its PK data, especially for those with super potent NMEs. As long as their potencies and mechanism are attractive, they should be tested in in vivo efficacy models despite poor PK profiles. Indeed, a number of marketed drugs have shown poor PK profiles but have still become the top ever selling drug - Lipitor (Atorvastatin), with absolute bioavailability of $5 \%$ and $14 \%$ in rat and human, respectively $[10,11]$. As illustrated in Figure 3 , in an analysis of 600 market drugs [12], more than $30 \%$ of them have relatively low bioavailability ( $F<30 \%$ ), and $22 \%$ of the drugs have even lower bioavailability ( $F<10 \%$ ). Nearly $50 \%$ of the drugs have moderate-to-high clearance, and $17 \%$ show high clearance, or extremely high clearance (even over blood flow).

As mentioned above, some of these top selling drugs have many undesirable properties in terms of physicochemical properties, such as solubility, Log D, in vitro microsomal stability (Clint), and permeability/efflux data, as well as high in vivo clearance or low bioavailability. If we use only PK data to screen the compounds, some potential drug-like compounds might be discarded. In other words, the Atorvastatin-like compounds would never have become good drugs from the sole view of PK profiles. These examples suggest that a better understanding of in vitro-in vivo correlation and frontloading PK/PD is crucial for compound selection even at an early stage. Obviously, a super PD can save a poor PK as long as the PD effect is notable. 


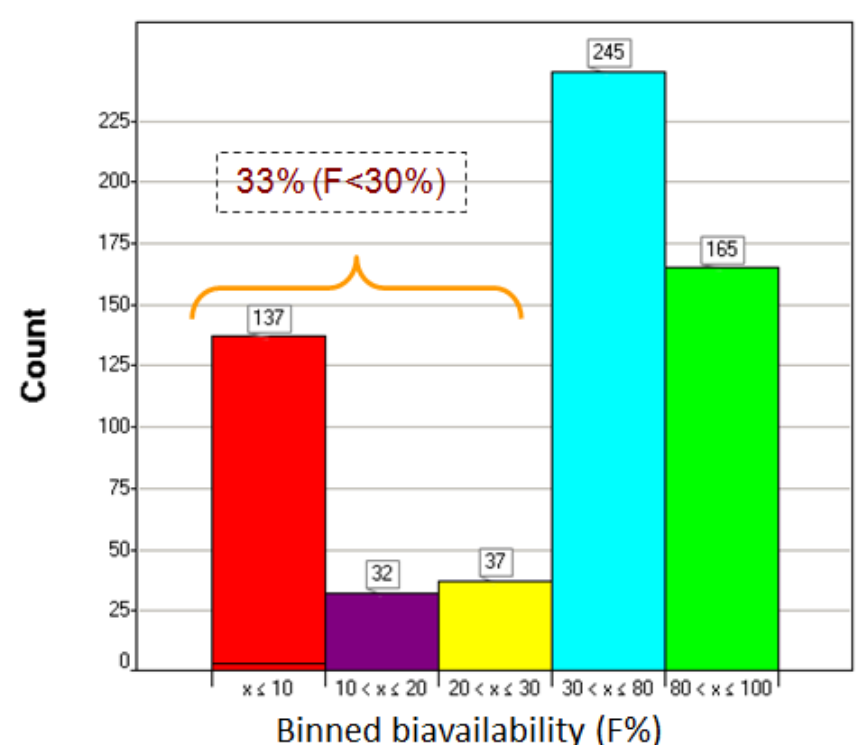

Binned biavailability (F\%)

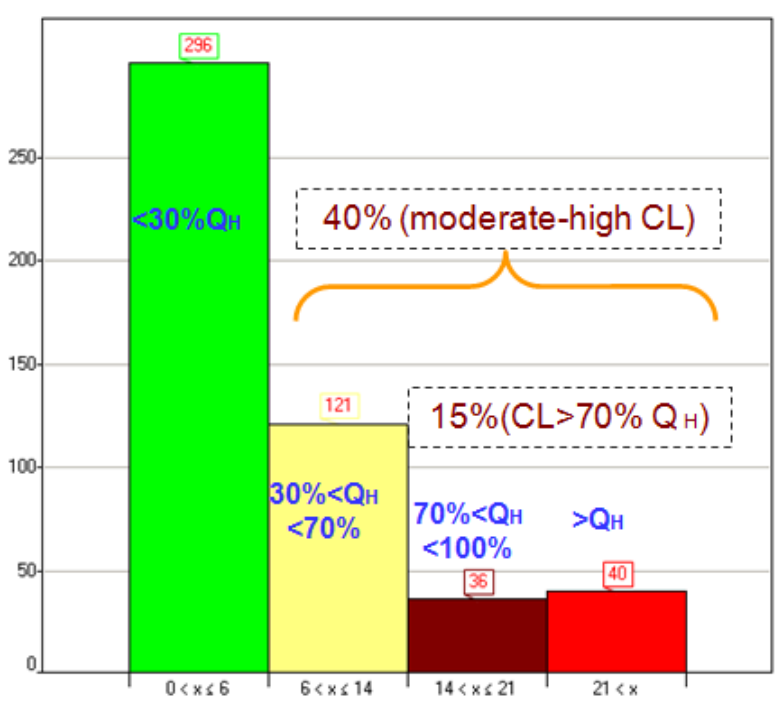

Binned clearance $\mathrm{CL}(\mathrm{ml} / \mathrm{min} / \mathrm{kg})$

Figure 3: Distribution of human clearance and bioavailability of market drugs

\section{Concluding remarks}

Drug discovery and development are a high-risk, high-cost but highly rewarding business. Many resources are invested, and thus wasted on, candidate products that are subsequently found to have unacceptable profiles when evaluated in human - only less than $10 \%$ of INDs from NMEs progress beyond the submission of new drug application (NDA) according to the FDA's report [8]. As a very comprehensive and important science and technology platform bridging efficacy and safety, DMPK has become a discovery function to optimise ADME properties in drug design, to reduce attrition rates. Recent studies have demonstrated that it is viable to implement high-throughput cutting-edge technologies and cost-effective ADME/DMPK screening assays, and design relevant studies to address various ADME issues at different stages for de-risking the investment and avoiding development mistakes early.

Apparently, at drug discovery stage, a better understanding of SAR and in vitro-in vivo correlations, and more input on drug design can be anticipated. Moreover, frontloading PK/PD for a better understanding of the efficacy-exposure relationship and dose prediction as early as possible can benefit projects. The integration of in vitro and in vivo drug metabolism data with physicochemical properties as well as PK and PD data is highly recommended for safety and toxicity liability evaluation, e.g., are there any drug accumulations? Are there any potential toxic metabolites? Are there any likely DDIs? Are estimated safety margins enough? These safety-related questions have to be answered as early and clearly as possible to de-risk the drug discovery projects. Another concern is risk assessment associated with hERG, CYP inhibition and induction, metabolite profiling and safety, transporter substrate and inhibition, safety margin, etc., which is of ultimate importance for CS and decision-making for the IND-enabling package. Future ADME studies will continue to focus on P450 enzymes and transporters' impact on safe drug delivery as well as accurate prediction of human PK, PK/PD modeling and optimum clinical dosage.

On the other hand, from the point of view of regulatory review, the FDA has been becoming increasingly conservative than ever, except for oncology and life-threatening diseases where a risk-benefit assessment can be considered. If a drug is toxic at clinically relevant doses, the IND may never go forward. Therefore, a complete study/data package including as much detailed toxicity information as possible can facilitate IND filing and increase the clinical trial success rate, which should cover pharmacology (efficacy and animal 
proof of principle) studies, a rodent general toxicology study of at least a 14-day duration, a non-rodent general toxicology study of at least a 14-day duration, genotoxicity studies, safety pharmacology studies, ADME/PK as well as bioanalytical validations, etc. In the end, we must clearly answer these two key questions:

(1) Is the preclinical candidate safe enough to take into humans?

(2) Is the preclinical candidate effective in patients?

Acknowledgements: The author would like to thank Dr. Kin Tam for proofreading this article with valuable comments.

Declaration: This article reflects the author's personal opinions. The author declares that he has no competing financial interests.

\section{References}

[1] FDA: Draft Guidance for Industry: Drug Interaction Studies - Study Design, Data Analysis, Implications for dosing, and Labeling Recommendation, (Sept. 2006)

[2] FDA: Guidance for Industry: Drug Interaction Studies - Study Design, Data Analysis, Implications for dosing, and Labeling Recommendation (February 2012)

[3] FDA: Guidance for Industry: Safety testing of drug metabolites (February 2008)

[4] ICH M3(R2): Guidance on nonclinical safety studies for the conduct of human clinical trials and marketing authorization for pharmaceuticals (June 2009)

[5] FDA: Guidance for Industry S7B Nonclinical Evaluation of the Potential for Delayed Ventricular Repolarization (QT Interval Prolongation) by Human Pharmaceuticals (2005)

[6] FDA: Guidance for Industry Bioanalytical Method Validation (2001)

[7] FDA: Guidance for Industry Content and Format of Investigational New Drug Applications (INDs) for Phase 1 Studies of Drugs, Including Well-Characterized, Therapeutic, Biotechnology-derived Products (1997)

[8] FDA: Guidance for Industry, Investigators, and Reviewers - Exploratory IND Studies (Jan 2006)

[9] G. Lappin, M. Seymour, Bioanalysis 2 (2010) 1315-1324.

[10] Y.Y. Lau, H. Okochi, Y. Huang and L.Z. Benet, Drug Metabolism and Disposition 34 (2006) 1175-1181.

[11] H. Lennernas, Clinical Pharmacokinetics 42 (2003) 1141-1160.

[12] L.S. Goodman, A. Gilman, The Pharmacological Basis of Therapeutics, McGraw-Hill Publishers, New York, USA, 2006.

$\begin{array}{ll}\text { Abbreviations } & \\ \text { ADME } & \text { Absorption Distribution Metabolism Excretion } \\ \text { CRO } & \text { Contact Research Organization } \\ \text { DMPK } & \text { Drug Metabolism and Pharmacokinetics } \\ \text { PK } & \text { Pharmacokinetics }\end{array}$




\begin{tabular}{ll} 
CYP450 & Cytochrome P450 \\
MDCK & Madin-Darby canine kidney cells \\
PPB & Plasma Protein Binding \\
hERG & human Ether-a-go-go Related Gene \\
MetID & Metabolite Identification \\
PD & Pharmacodynamics \\
P-gp & P-glycoprotein \\
MTD & Maximum Tolerated Dose \\
IND & Investigational New Drug \\
GLP & Good Lab Practice \\
CL & Clearance \\
F & Absolute bioavailability \\
Ce(ss) & Effective plasma concentration at steady stage \\
$\tau$ & Dose interval \\
CS & Candidate Selection \\
PMAPA & Parallel Artificial Membrane Permeability Assay \\
NMEs & New Molecular Entities \\
& \\
\hline &
\end{tabular}

(C2013 by the authors; licensee IAPC, Zagreb, Croatia. This article is an open-access article distributed under the terms and conditions of the Creative Commons Attribution license (http://creativecommons.org/licenses/by/3.0/) (cc) EY 